rensvoraussetzung sei. Daß das Verfahren rechtsstaatlich und "fair" zu sein habe, wird nun zwar nicht bestritten werden können, es kennzeichnet die Summe rechtsstaatlich fundierter Einzelvorschriften ebenso wie die Verhaltensanforderungen an Gericht und Staatsanwaltschaft; daß die dogmatische Kategorie der Verfahrensvoraussetzung dieser Aussage gerecht wird, läßt sich nur schwer vertreten.

IV. Verfahrenshindernisse, so läßt sich nach allem etwas vergröbernd sagen, sind einigermaßen exakt beschreibbare und beschriebene Ereignisse ${ }^{29}$, die mit der (unterschiedlich begründeten) Aussage verbunden sind, daß eine Sachentscheidung ausgeschlossen ist. Hierauf genau ist ihre Feststellung und ihre Konsequenz zugeschnitten. Sie festzustellen bedarf es keiner Hauptverhandlung, das Mittel hierfür ist der Freibeweis ${ }^{30}$. Sie entlasten den Schuldigen, weil sie ihm die Verurteilung ersparen, aber sie belasten den Unschuldigen, weil sie ihn um die Chance der positiven Sachentscheidung bringen.

Die Rechtsstaatswidrigkeit des Verfahrens, der Verstoß gegen das Fairneß-Gebot ist von anderer Qualität. Bei Verstoß gegen rechtsstaatlich verankerte Prozeßnormen steht ein differenziertes Rechtsschutz- und Reaktionssystem zur Verfügung; es reicht prozessual - wie anhand des hier besprochenen Falles gezeigt werden konnte - vom Ablehnungsrecht über Beweisverbote bis zur Revisibilität, es kann materiell-rechtlich sowohl bei der überlangen Verfahrensdauer ${ }^{31}$ als auch bei der Tatprovokation $^{32}$ als Strafmilderungsgrund berücksichtigt werden. Es mag mühsamer sein, es ist aber der Sache dienlicher, sich in diesem differenzierten Reaktionssystem zu bewegen, als das Institut der Verfahrenshindernisse für eine Aufgabe einzusetzen, für die es keine adäquate Ausgestaltung bieten kann.

$\mathrm{Ob}$ in äußersten Extremfällen rechtsstaatswidrige Rechtsverstöße irreparabler Art von solchem Schweregrad vorkommen können, daß es gerechtfertigt sein kann, auf die Durchsetzung des Sanktionsanspruchs auf die Dauer gänzlich auch dort zu verzichten, wo das geltende Recht nicht, wie in $\$ 60 \mathrm{StGB}$ oder $\$ 153 \mathrm{StPO}$, eine ausreichende Ermächtigung für einen solchen Verzicht enthält, muß mit der Skepsis gegen das Verfahrenshindernis schwerwiegender Rechtsstaatswidrigkeit, auch wenn es in konkreten Spielarten auftritt, nicht notwendig verneint werden. Nur sollte ein solches aus der Verfassung (vielleicht) ableitbares „Verfolgungsverbot" nicht auf das dogmatische Gebilde Verfahrenshindernis zurückgreifen, bei dem die Verlockung zu vorschnellem und undifferenziertem Einsatz besonders groß ist, weil es in Voraussetzungen und Folgen einigermaßen klar umrissen und vom Gesetzgeber partiell anerkannt ist. Es ist eine bisher kaum in Angriff genommene Aufgabe für Rechtsprechung und Strafprozeßwissenschaft, außerhalb des Begriffs des Verfahrenshindernisses Voraussetzungen, Folgen und Standort eines äußerstenfalls in Betracht $\mathrm{zu}$ ziehenden „Verfolgungsverbots“ schwerwiegender Rechtsstaatswidrigkeit selbständig zu entwickeln, wenn sich zeigen sollte, daß das vorhandene Reaktions- und Rechtsschutzsystem nicht ausreicht.

\footnotetext{
29 Insoweit steckt in der Auffassung, daß Umstände ausscheiden, die überwiegend Gegenstand wertender Beurteilung sind, ein richtiger Kern; in ähnliche Richtung wohl Volk (o. Fn. 22), S. 216.

30 So jedenfalls die Rechtsprechung und ein erheblicher Teil des Schrifttums; vgl. m. w. N. LR-Gollwitzer, 24. Aufl., 1985, \244 Rdn. 3 ff.

31 BGHSt. 21, 83; 24, 293; 27, 274; BGH NStZ 1982, 291

32 BGH NStZ 1982, 126; StrVert. 1982, 221; ausführlich BGHSt. 32, 345 ff. = NJW 1984, 2302.
}

\section{Sittenwidrigkeit und Abstraktion}

\section{Von Professor Dr. Reinhard Zimmermann, Cape Town/ Südafrika}

Sind Verfügungen, insbesondere Übereignungen, abstrakt oder kausal? Diese Frage liegt im Schnittpunkt zwischen Schuld- und Sachenrecht; die Antwort, die eine Rechtsordnung gibt, hat zwar nicht für das praktische Ergebnis, wohl aber für Denkansatz, Lösungsmethode und die relative Bedeutung des dogmatischen Institutioneninstrumentariums Bedeutung. Dem BGB liegt das Abstraktionsprinzip zugrunde. Das wird nirgends bestritten und gilt weltweit geradezu als eines der Charakteristika der deutschen Kodifikation ${ }^{1}$. Seine rechtspolitische Zweckmäßigkeit wurde eine Zeitlang als immer fragwürdiger empfunden, und insbesondere während der dreißiger und vierziger Jahre kam es zu scharfen Attakken, die in der Forderung nach seiner Beseitigung gipfelten $^{2}$. Inzwischen hat sich die Debatte soweit beruhigt, daß in Kommentaren und Lehrbüchern Konsequenzen und Einschränkungen des Abstraktionsprinzips registriert, aber nicht mehr grundsätzlich in Frage gestellt werden. Bei einer Durchsicht der gängigen Lehrmeinungen ergeben sich freilich eine Reihe von Fragen und Widersprüchlichkeiten.

Die größten Schwierigkeiten bereiten seit jeher die sogenannten Wirksamkeitsgrenzen des Abstraktionsprinzips. Dabei lasse ich Konstruktionen wie die, die Wirksamkeit des Verpflichtungsgeschäfts sei als Bedingung oder als Geschäftsgrundlage der Verfügung oder aber beide Geschäfte seien als Teile eines einzigen Rechtsgeschäfts anzusehen, so daß bei Nichtigkeit des einen gemäß $\ 139$ BGB auch das andere nichtig sei, außer Betracht: sie setzen sich methodisch unzulässig über die Entscheidung des BGB-Gesetzgebers für das Abstraktionsprinzip hinweg. Interessant sind vielmehr die Fälle der Fehleridentität. Verpflichtungs- und Verfügungsgeschäfte sind je für sich auf ihre Wirksamkeit zu beurteilen; dabei kann sich freilich ergeben, daß beide unter demselben Mangel leiden und daher unwirksam sind. Das ist, soweit es etwa um die Frage der Geschäftsfähigkeit geht, unproblematisch. Sehr viel schwieriger ist die Frage, ob der Makel der Sittenwidrigkeit auf das Verfügungsgeschäft „durchschlagen“ kann. Hier drängt sich mitunter der Eindruck einer etwas prinzipienlosen Unsicherheit auf.

Verpflichtungen und Verfügungen sind Rechtsgeschäfte, die den im Allgemeinen Teil des BGB enthaltenen Regeln der Rechtsgeschäftslehre unterliegen. Sittenwidrige Rechtsgeschäfte sind nichtig; das gilt insbesondere auch für Fälle des Wuchers. Wucher wird im BGB als Spezialfall der Sittenwidrigkeit angesehen ${ }^{4}$. Man

\footnotetext{
$1 \mathrm{Vgl}$. Zweigert/Kötz, Einführung in die Rechtsvergleichung auf dem Gebiete des Privatrechts, Bd.I, 1971, S. $213 \mathrm{ff}$.

2 Vgl. bes. Heck, Das abstrakte dingliche Rechtsgeschäft, 1937; H. Brandt, Eigentumserwerb und Austauschgeschäft - Der abstrakte dingliche Vertrag und das System des deutschen Umsatzrechts im Licht der Rechtswirklichkeit, 1940.

3 Repräsentativ etwa Flume, Allgemeiner Teil des Bürgerlichen Rechts, 2. Band, 3. Aufl., 1979, \$12 III 4.

${ }_{4}$ Zur Gesetzgebungsgeschichte des $\$ 138$ II BGB vgl. neuerdings Luig, Vertragsfreiheit und Äquivalenzprinzip im gemeinen Recht und im BGB, in: Aspekte europäischer Rechtsgeschichte, Festgabe für Helmut Coing, 1982 (Ius Commune, Sonderheft 17), S. $171 \mathrm{ff}$. mit reicher Literaturübersicht, S. $204 \mathrm{ff}$.; zusätzlich Zimmermann, Richterliches Moderationsrecht oder Totalnichtigkeit? Die rechtliche Behandlung anstößig-übermäßiger Verträge, 1979, S. 142 ff., 153 m.w.N.
} 
sollte deshalb erwarten, daß hinsichtlich der Wirksamkeit von Verfügungsgeschäften, was wucherische und was sonst sittenwidrige Verträge betrifft, dieselben Regeln gelten. Das scheint jedoch nicht so zu sein. Eines der wenigen bei der Diskussion um die Reichweite des Abstraktionsprinzips seit jeher und einhellig anerkannten positivrechtlichen Argumente stützt sich auf den Wortlaut des $₫ 138$ II BGB: aus der Formulierung „versprechen oder gewähren läßt" sei zu schließen, daß, anders als bei sittenwidrigen Geschäften gemäß $\$ 138$ I BGB, im Falle des Wuchers nicht nur das Verpflichtungsgeschäft, sondern auch das Erfüllungsgeschäft des Bewucherten nichtig sei ${ }^{5}$.

Dies Argument ist nicht unbedingt zwingend. Der heutige $\$ 138$ II BGB wurde erst durch die XII. Kommission, an die der Reichstag den Entwurf des BGB überwiesen hatte, in das Gesetzbuch eingefügt. Über die Alternative des "gewähren-Lassens" hat man sich ausweislich der Materialien keine näheren Gedanken gemacht: ${ }^{6}$ man übernahm die Formulierung vielmehr aus den Wuchervorschriften des StGB. Sie geht zurück auf das Gesetz, betreffend den Wucher vom 24.5.18807. $\$ 302 \mathrm{a}$, der durch dessen Art. 1 in das Strafgesetzbuch aufgenommen wurde, lautete: „Wer unter Ausbeutung der Nothlage, des Leichtsinns oder der Unerfahrenheit eines Anderen für ein Darlehn oder im Falle der Stundung einer Geldforderung sich oder einem Dritten Vermögensvortheile versprechen oder gewähren läßt, welche den üblichen Zinsfuss dergestalt überschreiten..." Maßgeblich für die hier interessierende Alternativformulierung war die Vermeidung von Strafbarkeitslücken. „Sich gewähren lassen" bezog sich in diesem Zusammenhang offenbar nicht auf die Leistung bereits versprochener Vermögensvorteile, bedeutete demnach nicht "sich ein Versprechen erfüllen lassen“. War schon das ",sich-versprechen-Lassen" strafbar, so bestand für den Gesetzgeber kein Anlaß, zusätzlich die Geltendmachung des Versprechens unter Strafe zu stellen. Das „sich-gewähren-Lassen“ ist damit auf die Gewährung nicht vorher versprochener Vermögensvorteile zu beziehen; durch die Aufnahme dieser Alternative in das Gesetz sollte sichergestellt sein, daß die Strafbarkeit sich auf diejenigen Darlehnsgeber erstreckt, die die wucherischen Vermögensvorteile sogleich bei Eingehung des Darlehensgeschäftes, ohne vorheriges Versprechen, empfangen haben. In diesem Sinne wurde $\$ 302$ a StGB in den ersten Jahren nach seiner Aufnahme in das Strafgesetzbuch allenthalten verstanden $^{8}$, und auch heute wird in der strafrechtlichen Literatur darauf hingewiesen, daß die Annahme der wucherischen Vorteile bei vorausgegangenem Versprechen gegenüber der Entgegennahme dieses Versprechens keine selbständige Bedeutung habe, sondern nur die Fortentwicklung der Vertragsberechtigung bedeute? ${ }^{9}$. In der Tat: wer die Vorteile zunächst versprochen hat, wird, wenn er leistet, nicht abermals bewuchert. Er übergibt den Wucherzins solvendi causa, um die (unwirksam) eingegangenen Verpflichtung zu erfüllen. - Daß mit den Worten „oder (sich) gewähren läßt", als man sie in das Zivilrecht übernahm und mit der Nichtigkeitssanktion verknüpfte, etwas anderes und weitergehendes gemeint sein sollte, ist nicht ersichtlich. Zudem spricht nichts dafür, daß in $\$ 138$ II BGB, was die Wirksamkeit des abstrakten Erfüllungsgeschäfts angeht, von dem unter dem Reichswuchergeset $z^{10}$ anerkannten Rechtszustand abgewichen werden sollte. Erfaßt werden, so gesehen, von der Nichtigkeit auch Fälle, in denen der Leistung kein verpflichtender Vertrag vorausgegangen ist ${ }^{11}$, sondern in dem diese entweder mit dem Abschlusse eines solchen zeitlich zusammenfällt oder aber auch lediglich von einer Abrede über den Rechtsgrund begleitet wird, ohne die die Leistung nicht rechtsbeständig wäre und bereicherungsrechtlich rückabgewickelt werden müßte. Da diese Rechtsgrundabrede freilich hier gerade unwirksam ist (aber auch hier nur diese!) kann kondiziert, aber nicht vindiziert werden.

Als ebenfalls nicht zwingend erscheint ein anderes, insbesondere früher häufig vorgebrachtes positivrechtliches Argument, diesmal zu Stützung des Abstraktionsprinzips. Gebe das Gesetz in $\$ 817$ BGB bei sittenwidrigen Leistungen nur eine Kondiktion, so setze es die Wirksamkeit des Verfügungsgeschäftes voraus. Wäre die Übereignung nichtig, so wäre insbesondere schwer verständlich, daß $\$ 817,2$ BGB die Rückforderung bei eigener Unsittlichkeit des Gebers ausschließt, da dieser ja vindizieren könnte ${ }^{12}$. Die Vorschrift des $\$ 817$ BGB ist dem Gesetzgeber anerkanntermaßen mißlungen ${ }^{13}$ und bietet sich somit als Ausgangspunkt für derartige Deduktionen nicht an. $\$ 817,1 \mathrm{BGB}$ ist neben der allgemeinen Kondiktion des $\$ 812$ BGB sowieso gegenstandslos ${ }^{14}$ oder hat allenfalls einen nur drei Fallgruppen umfassenden, wenig bedeutenden eigenständigen Anwendungsbereich $^{15}$. $\$ 817,2$ BGB, nach seinem Wortlaut auf $\$ 817,1$ BGB bezogen und damit ebenfalls in Gefahr (so gut wie) gegenstandslos zu sein, wird jedoch einerseits auch auf die Leistungskondiktion des $\$ 812$ BGB angewandt ${ }^{16}$, muß andererseits aber auch auf den Herausgabean-

RGZ 57, 95 (97); 109, 201 (202); BGH WM 1974, 774; Erman/Brox, BGB 7. Aufl., 1981, \138, Rdn. 24; Jauernig, BGB, 2. Aufl., 1981, \138, 5 a; Münchener Kommentar/Mayer-Maly, Bd. 1, 1978, S 138, Rdn. 137; Palandt/Heinrichs, BGB 43. Aufl., 1984, $\$ 138,4$ b; Soergel/Hefermehl, BGB, Bd. 1, 1978, $\$ 138$ Rdn. 49; Staudinger/H. Dilcher, BGB, 12. Aufl., 1979, \$138, Rdn.111; Flume (o.A. 3), $₫ 18,7 \mathrm{~d}$; Larenz, Allgemeiner Teil des deutschen Bürgerlichen Rechts, 6. Aufl., 1983, \\$22 III d; Medicus, Allgemeiner Teil des BGB, 1982, Rdn. 712.

6 Vgl. etwa Kommissionsbericht, in: Mugdan, Die gesamten Materialien zum Bürgerlichen Gesetzbuch für das Deutsche Reich, I, 1899, S. 969 f.

RGBl. $109 \mathrm{ff}$; vgl. dazu etwa Nr. 265 in Stenogr. Ber. RT, 4. Lp., II. Session, 1879,6 . Bd. und Nr. 58 in Stenogr. Ber. RT, 4. Lp., III. Session, 1880, 3. Bd.

Vgl. RGSt. 4, 109 (110 f.); RGRspr. 3, Nr. 408 (S. 846, 848); von Olshausen Kommentar zum Strafgesetzbuch für das Deutsche Reich, 6. Aufl., 2.Bd., 1901, $\$ 302$ a, 6; G. Freudenstein, Das Reichs-Wuchergesetz, 1882, S. 39 ff.

Vgl. Leipziger Kommentar/von Bubnoff, StGB, 10.Aufl., 1980, $\$ 302$ a, Rdn. 54; Schönke/Schröder/Stree, StGB, 21. Aufl., 1982, \302 a, Rdn. 19.

10 Art. 3 des Gesetzes vom 24.5.1880 in der Fassung des Art. II des Gesetzes vom 19.6.1893, RGBl. 197 ff.; hierzu v. a. C. Raiser, Die privatrechtliche Behandlung des Wuchers nach den Reichsgesetzen vom 24.5.1880 und 19.6.1893 und im Bürgerlichen Gesetzbuch, Diss. Tübingen, 1898.

1 Das war im Gemeinen Recht jedenfalls theoretisch die Ausnahme. Das Darlehen war rechtstechnisch ein unentgeltliches Geschäft und außerdem ein Realvertrag; es kam erst mit der Übergabe der Darlehensvaluta zustande. Vorausgehen konnte ein pactum de mutuo dando mit Zinsversprechen. Demgemäß hieß es in dem Antrag von Kleist-Retzow, von Flottwell, Freiherr von Marschall (Nr. 55 in Stenogr. Ber. RT, 4. Lp., II. Session, 1879, 4. Bd.), auf den das Reichswuchergesetz zurückgeht, auch sehr plastisch: wer sich (...) übermäßige Vorteile „vorbedingt oder gewähren läßt".

12 Vgl. etwa Enneccerus/Nipperdey, Allgemeiner Teil des Bürgerlichen Rechts, 15. Aufl., 1959/60, $\$ 191$ II 3 (Fn. 28).

13 Vgl. z. B. Alternativkommentar zum BGB/Joerges, Bd.3, 1979, $\$ 817,1$; Staudinger/Lorenz, BGB, 12. Aufl., 1979, \$817, Rdn. 1 ff., 10; Enneccerus/Lehmann, Recht der Schuldverhältnisse, 15. Aufl., 1958, \$226, 4 (S.908); Larenz, Lehrbuch des Schuldrechts, Besonderer Teil, 12. Aufl., 1981, \$69 III b.

${ }^{14}$ So H.Honsell, Die Rückabwicklung sittenwidriger oder verbotener Geschäfte, 1974, S. 10 f., 32 ff.; Erman/H. P. Westermann, \$817, Rdn. 6.

15 Enneccerus/Lehmann, \$226, 1; Larenz (o. A. 13), 69 III a; Medicus, Bürgerliches Recht, 11. Aufl., 1983, Rdn. 694; Koppensteiner/Kramer, Ungerechtfertigte Bereicherung, 1975, S.73; Reeb, Grundprobleme des Bereicherungsrechts, 1975, S. 64 f.; vgl. weiterhin Münchener Kommentar/Lieb, Bd. 3/2, 1980, \$817, Rdn. 4 ff.; Staudinger/Lorenz, 12. Aufl., 1979, \$817, Rdn. 6 ff.

16 RGZ 151, 70 (71 ff.); 161, 52 (55); BGHZ 36, 395 (399); 50, 90 (91); BAG BB 1963, 348; Erman/H.P. Westermann, \$817, Rdn. 11; Jauernig/Schlechtriem, $\$ 817,5$; Palandt/Thomas, $\$ 817,1$ a; Staudinger/Lorenz, $\$ 817$, Rdn. 10; Fikentscher, Schuldrecht, 6. Aufl., 1976, \$99 III 4 c; Flume (o. A. 3) \$18, 10 a; Larenz (o. A. 13), $\$ 69$ III b; vgl. auch Münchener Kommentar/Lieb, $\$ 817, \mathrm{Rdn} .10 \mathrm{ff}$. 
spruch gemäß $₫ 985$ BGB erstreckt werden ${ }^{17}$. Damit fällt das Argument in sich zusammen.

Man sollte sich deshalb bei der Rechtsanwendung nicht von zufälligen Formulierungen des Gesetzes beeinflussen lassen, sondern Konsistenz und Folgerichtigkeit der Durchführung des diesem zugrundeliegenden Regelungsprinzips in den Vordergrund der Betrachtung rükken. Dies ist nun zweifellos das Abstraktionsprinzip. Der BGB-Gesetzgeber rezipierte ${ }^{18}$ die maßgeblich von Friedrich Carl von Savigny geprägte ${ }^{19}$, in der Pandektistik weiter ausgebaute und in der zweiten Hälfte des 19. Jahrhunderts herrschende Lehre vom dinglichen Vertrag als einem inhaltlich und funktional abstrakten „Formalakt ${ }^{\text {"20: }}$ inhaltlich abstrakt, da nur die beiden auf Übertragung des Eigentums gerichteten Willenserklärungen umschließend und insofern zuwendungszweckfrei ${ }^{21}$, funktional abstrakt, da in seiner Geltung losgelöst von der Gültigkeit des zugrundeliegenden Schuldverhältnisses. Von diesem Ausgangspunkt her ist es sicherlich folgerichtig, wenn als Regel davon ausgegangen wird, Verfügungsgeschäfte würden von der Sittenwidrigkeit zugrundeliegender Verpflichtungsgeschäfte nicht tangiert. Freilich glaubt man weithin, Ausnahmen einräumen zu müssen.

So erfaßt nach Ansicht der Rechtsprechung die Sittenwidrigkeit das Erfüllungsgeschäft dann, wenn gerade in ihm der Sittenverstoß liegt oder mit ihm sittenwidrige $Z$ wecke verfolgt werden ${ }^{22}$. Das ist angenommen worden etwa in einem Fall, in dem ein Ehemann, um seine Ehefrau zur Einreichung der Klage auf Ehescheidung zu bewegen, ihr im Rahmen eines Unterhaltsvertrages für den Fall der Ehescheidung ein Grundstück übertragen hatte ${ }^{23}$ : nicht nur der Unterhalts- und Auseinandersetzungsvertrag sei sittenwidrig, sondern auch die Grundstücksübereignung. Demgegenüber ist jedoch darauf hinzuweisen, daß die Übereignung eines Grundstückes unter Ehegatten nicht anstößig ist. Nur darauf kommt es jedoch an; das dingliche Rechtsgeschäft ist losgelöst von dem Zuwendungszweck zu würdigen und hat lediglich die Übertragung des Grundstückseigentums zum Gegenstand. Diese Rechtsänderung als solche ist ebenso makelfrei wie die Übereignung eines Geldscheines an eine Prostituierte, um ein Beispiel heranzuziehen, in dem nach wohl allgemeiner Ansicht die Sittenwidrigkeit sich auf das Verpflichtungsgeschäft beschränken soll. Auch bei einer Zession an einen vermögenslosen Erwerber in der Absicht, dem Prozeßgegner die Kostenerstattung unmöglich zu machen ${ }^{24}$, ist nicht ersichtlich, wieso gerade in der Zession der Sittenverstoß liegen soll. Sittenwidrig ist das Verpflichtungsgeschäft, die Zession an sich ist nicht zu beanstanden, auch wenn der Zessionar arm ist. Es ist der mit der Zuwendung verfolgte Zweck, der das Geschäft in einem trüben Licht erscheinen läßt; dieser ist aber bei der Würdigung außer Betracht zu lassen. Gleiches gilt, um einen weiteren Fall zu nennen, an den bei der erwähnten Ausnahme gedacht ist ${ }^{25}$, für das Erfüllungsgeschäft des Wucherers ${ }^{26}$ : die Übereignung ist sittlich nicht zu mißbilligen; es ist die Tatsache, daß der Wucherer unter Ausnutzung bestimmter subjektiver Gegebenheiten sich in einem auffälligen Mißverhältnis zur Leistung stehende Vermögensvorteile versprechen läßt, die als sittenwidrig beurteilt werden. Aus diesem Grund ist das der Darlehenshingabe zugrunde liegende Geschäft unwirksam. Die von der Rechtsprechung entwickelte Formel erscheint damit zur Begründung der vom Abstraktionsprinzip zugelassenen Ausnahmen als nicht geeignet. Erweitert man sie in dem Sinne, daß sie Fälle wie die erwähnten erfaßt, wird sie zu weit und unsicher und hebt geradezu das Abstraktionsprinzip aus den Angeln: bezieht man nämlich den Zweck der Zuwendung in die rechtliche Würdigung mit ein, ist nicht deutlich, wo dann noch Grenzen gezogen werden und Verfügungsgeschäfte als nicht nichtig beurteilt werden können.

In der Literatur wird demzufolge auch nach anderen Kriterien gesucht. Dingliche Erfüllungsgeschäfte, so eine nicht selten vertretene Ansicht, seien sittenwidrig, wenn der Sittenverstoß gerade im Verhalten gegenüber dem Kontrahenten liege oder ein Interesse Dritter oder das Gemeininteresse tangiert werde ${ }^{27}$. Prototyp der ersten Fallgruppe ist das Erfüllungsgeschäft des Bewucherten. Aber auch hier gilt: die Übereignung einer bestimmten Summe Geldes ist nicht per se sittenwidrig; erst, wenn in Rechnung gestellt wird, daß dies Geld als Zins für ein Darlehen zu bezahlen ist, hinsichtlich dessen die Voraussetzungen des $\$ 138$ II $\mathrm{BGB}$ erfüllt sind, läßt sich ein derartiges Urteil fällen. Auch verdeckte übermäßige Sicherungsgeschäfte etwa in Form von Sicherungsübereignungen mit dem Zwecke, Dritte über die Kreditwürdigkeit des Schuldners zu täuschen oder um den Schuldner auszusaugen, sind gemäß $\ 138$ I BGB sittenwidrig ${ }^{28}$. Dies Verdikt muß sich aber auf das Verpflichtungsgeschäft beschränken, denn die Übereignungen an sich können, auch wenn sie noch so große Werte erfassen, unter anderen Umständen durchaus der Erfüllung eines sittenkonformen Vertrages dienen. Ihnen ist die Sittenwidrigkeit, die sich aus den Umständen (der Lage und den Vermögensverhältnissen des Schuldners) und den von den Parteien verfolgten Zwecken ergibt, nicht anzusehen. Soweit davon ausgegangen wird, daß die Wirksamkeit des Verfügungsgeschäftes in derartigen Fällen sich mit dem Abstraktionsprinzip vereinbaren läßt, da die Sittenwidrigkeit hier selbständig für das abstrakte Zuwendungsgeschäft gegeben $\mathrm{se}^{29}$, kann dem somit nicht gefolgt werden. Soweit die von der erwähnten Formel erfaßten Fälle als Ausnahmen vom Abstraktionsprinzip beschrieben werden ${ }^{30}$, sind sowohl die mangelnde

17 Anders die Rechtsprechung: RG LZ 1923, 565 (566f.); 1924, 329; OGHZ 4, 57 (65); BGH NJW 1951, 643; BGH LM Nr. 20 zu $\$ 817$; vgl. auch BGHZ 63 , 365 (369). Aus der Literatur vgl. etwa: Palandt/Bassenge, Einf. v. $\$ 854,5 \mathrm{a}, \mathrm{cc}$ Münchener Kommentar/Lieb, $\$ 817$, Rdn. 20; Staudinger/Lorenz, $\$ 817$, Rdn. 14; Baur, Lehrbuch des Sachenrechts, 12. Aufl., 1983, \ 5 IV 3 a a. E.; Larenz (o. A. 13), $\$ 69$ III b; Medicus (o.A. 15), Rdn.697; Wolff/Raiser, Sachenrecht, 10. Aufl., 1957, \$84 IV 4; Koppensteiner/Kramer (o.A. 15), S.76; von Caemmerer, SJZ 1950,650

${ }^{18}$ Vgl. Motive, in Mugdan III, S. 4 f.; vgl. auch Brandt (o. A. 2), S. $116 \mathrm{ff}$

19 Eingehend hierzu Felgentraeger, Friedrich Carl v. Savignys Einfluß auf die Übereignungslehre, 1927, bes. S. $24 \mathrm{ff}$.

20 Windscheid/Kipp, Lehrbuch des Pandektenrechts, 9. Aufl., 1906, $\$ 172,6$ (Fn. 16 a); vgl. im übrigen Brandt (o. A. 2), S.67 ff.; Ranieri, in: Wissenschaft und Kodifikation des Privatrechts im 19. Jahrhundert, Bd. III, 1977, S. $90 \mathrm{ff}$

${ }^{21}$ Vgl. etwa von Tuhr, Der Allgemeine Teil des Deutschen Bürgerlichen Rechts, Bd. II, 2, 1918, \$72 III (S. 80 f.); Beuthien, Zweckerreichung und Zweckstörung im Schuldverhältnis, 1969, S. 286; Jahr, AcP 168 (1968), 16

22 RGZ 109, 201 (202); RGZ 145, 152 (154); BGH WM 1966, 1221 (1223); Münchener Kommentar/Mayer-Maly, \$138, Rdn. 138; Soergel/Hefermehl, $\$ 138$, Rdn. 44.

3 RGZ 145, $152 \mathrm{ff}$; hierzu auch Flume (o. A. 3), $\$ 18,8 \mathrm{c} \mathrm{bb}$

24 RGZ 81, $175 \mathrm{ff}$.

25 Staudinger/H.Dilcher, $\$ 138, \mathrm{Rdn} .112$.

26 Seine Beurteilung ist in der Literatur freilich umstritten: vgl. Staudinger/ H. Dilcher, $\$ 138$, Rdn. 111; Enneccerus/Nipperdey (o. A. 12), \$192 III 1 m. w. N. aus der Rechtsprechung. Überwiegend wird für Wirksamkeit plädiert: Erman Brox, $\$ 138$, Rdn. 25; Jauernig, $\$ 138,5$ a; Soergel/Hefermebl, $\$ 138, R d n .49$; Flume (o. A. 3), \18, 7 d; Larenz (o.A. 5), \$22 III d a.E.

27 Flume (o. A. 3), \$18, 8 c bb; Larenz (o. A. 5), \$22 III c; Honsell (o. A. 14), S. 55; differenzierend Palandt/Heinrichs, $\$ 138,2 \mathrm{~d}, 3 \mathrm{c}$.

28 Vgl. z. B. Staudinger/H. Dilcher, $\$ 138$, Rdn. 44; Larenz (o. A. 5), $\$ 22$ III c.

29 Flume (o.A. 3), \18, 8c bb; ähnlich Honsell (o. A. 14), S. 55.

30 Vgl. die zutreffende Einordnung bei Münchener Kommentar/Mayer-Maly, $§ 138$, Rdn. 138 . 
Präzision dieser Ausnahmen zu beanstanden ${ }^{31}$ als auch insbesondere ihre zur Aushöhlung des Abstraktionsprinzips neigende Weite. Ganz überwiegend werden durch das sittenwidrige Handeln Interessen entweder des Kontrahenten oder bestimmter Dritter oder der Allgemeinheit verletzt; andere Schutzzwecke des $\$ 138$ BGB sind kaum denkbar. Ist der Verkauf von „Sexartikeln“ sittenwidrig $^{32}$, so deshalb, weil Grundsätze der Sozialmoral mißachtet werden. $\$ 138$ BGB dient insoweit der „Absicherung anerkannter Ordnungen "33 und schützt damit ein Gemeininteresse. Auch hier ließe sich, akzeptiert man einmal die referierte Auffassung, vertreten, je weiter die Sittenwidrigkeit greife, desto effektiver werde das Gemeininteresse geschützt bzw. es sei auch hier die rechtsgeschäftliche Regelung der Änderung des Rechtszustandes und nicht nur das rechtsgeschäftliche Handeln sittenwidrig. Gerade erst durch das Verfügungsgeschäft kommt der Käufer in den Besitz der pornographischen Publikation, entfaltet diese ihre dem Schutzzweck des $\$ 138$ BGB zuwiderlaufende Wirksamkeit. Gleiches gilt, um zwei beliebige andere Beispiele anzuführen, bei einem sittenwidrigen Praxisverkauu ${ }^{34}$ oder bei der Zimmervermietung an Prostituierte zu überhöhtem Entgelt ${ }^{35}$. Auch hier realisiert sich die Gefahr, vor der $\$ 138$ BGB schützen möchte, gerade erst durch den Rechtsübergang: der Erwerber kann die Praxis erst dann in einer die Belange der Klienten (d. h. Dritter) beeinträchtigenden Weise führen, wenn sie ihm übertragen worden ist, die Prostituierte wird an die Prostitution dadurch gebunden, daß sie in wirtschaftlicher Abhängigkeit gehalten wird und über ihr Geld, nach der Übereignung desselben, nicht mehr selbst verfügen kann. Auch hier läßt sich, akzeptiert man nur einmal die zugrundeliegende Prämisse, sagen, das Recht perhorresziere auch die Änderung der sachenrechtlichen Zuständigkeit ${ }^{36}$.

Übrigens findet sich in anderen Argumentationszusammenhängen nicht selten die die genannten Gesichtspunkte vereinfachend zusammenziehende Auffassung, in besonders schweren oder krassen Fällen der Sittenwidrigkeit schlage der Makel auch auf das Verfügungsgeschäft durch ${ }^{37}$. Diese Ansicht läuft darauf hinaus, von einem bestimmten Grad der Sittenwidrigkeit an das Abstraktionsprinzip unberücksichtigt zu lassen und entweder die Sittenwidrigkeit des Verpflichtungsgeschäfts auf die Verfügung zu erstrecken oder aber den Geschäftszweck bei der Beurteilung des Verfügungsgeschäftes mit zu berücksichtigen. Beides ist unzulässig. Darüber hinaus sind überhaupt derartige Abstufungen innerhalb der Sittenwidrigkeit sehr fragwürdig. $\$ 138$ BGB bezeichnet äußerste Grenzen der rechtsgeschäftlichen Privatautonomie und ist Ausfluß des Gebots der Selbstachtung des Rechts. Diese Grenzen hat der Gesetzgeber sehr weit gezogen und damit der selbstherrlich-schöpferischen Gestaltungsfreiheit der einzelnen am Rechtsverkehr beteiligten Individuen ein weites Feld eingeräumt. Geprüft wird, ob ein Rechtsgeschäft gegen das Anstandsgefühl aller billig und gerecht Denkenden verstöß $\mathrm{t}^{38}$ und in diesem Sinne anstößig ist. Der Vorwurf der Sittenwidrigkeit ist der schwerste Makel, der einem Rechtsgeschäft anhaften kann und damit per se ein Superlativ, der sinnvoll nicht weiter steigerungsfähig ist. Ebenso wie die Rechtsordnung es ablehnt, sich in die Beziehungen zweier sittenwidrig handelnder Parteien einzumischen und ihre Machtmittel einem Gauner gegen den anderen zur Verfügung zu stellen ${ }^{39}$, muß sie sich hier einer weiteren Bewertung sittenwidrigen Tuns enthalten. Geschäfte, die sittenwidriger als andere, ihrerseits zwar auch sittenwidrige, aber doch nur normal oder geringfügig sittenwidrige Rechtshandlungen sind, gibt es nicht ${ }^{40}$.

Die entscheidende Frage besteht somit insgesamt darin, ob man bereit ist, das Abstraktionsprinzip anzuerkennen oder nicht, nicht, welche Ausnahmen man zuzulassen gedenkt. Denn entweder wird der Willensakt, durch den die Rechtsübertragung verwirklicht wird, als abstrakt gedacht oder nicht. Die Fragwürdigkeit der theoretischen Begründung der herrschenden Lehre hat denn auch dazu Anlaß gegeben, bereits den Ansatzpunkt der herrschenden Lehre in Frage zu stellen. Verfügungsgeschäfte seien nicht deshalb unwirksam, weil sie abstrakt seien, sondern weil die Nichtigkeitssanktion für sie keine Geltung beanspruchen könne ${ }^{41}$. Das erscheint als unzutreffend, soweit a priori die Anwendbarkeit des $\$ 138$ BGB auf Verfügungsgeschäfte geleugnet werden soll. Für den dinglichen Vertrag gelten die allgemeinen Regeln der Rechtsgeschäftslehre ${ }^{42}$, mithin auch die vom Gesetzgeber in den Allgemeinen Teil des BGB gestellten Regeln über die Wirksamkeit und Unwirksamkeit von Rechtsgeschäften. Wenn damit andererseits gemeint ist, entscheidend sei die sittliche Indifferenz einer Rechtszuständigkeit, so ist dem zuzustimmen. Diese ergibt sich aber gerade aus der inhaltlichen Abstraktion des Erfüllungsgeschäftes: der Eigentumsübergang als solcher ist nur dann sittenwidrig und damit nichtig, wenn er unter allen denkbaren Umständen als anstößig erscheint und wenn keine Situation denkbar ist, in der er der Erfüllung eines sittenkonformen Grundgeschäftes dient ${ }^{43}$. Das Abstraktionsprinzip beschränkt damit für Verfügungsgeschäfte den Beurteilungshorizont des $\$ 138 \mathrm{BGB}$ : während die Sittenwidrigkeit eines Rechtsgeschäftes sich normalerweise aus einer Gesamtbeurteilung ergibt, für die der aus der Zusammenfassung von Inhalt, Motiv und Zweck zu entnehmende Gesamtcharakter des Vertrages maßgeblich ist ${ }^{44}$, gilt für Verfügungsgeschäfte der Maß-

31 So Münchener Kommentar/Mayer-Maly, \$138, Rdn. 138.

32 Vgl. hierzu Münchener Kommentar/Mayer-Maly, $₫ 138$, Rdn. 58.

33 Münchener Kommentar/Mayer-Maly, $\$ 138$, Rdn. 35 .

34 Hierzu etwa Staudinger/H.Dilcher, $\$ 138$, Rdn. 74, 76; Münchener Kommentar/Mayer-Maly, \$138, Rdn. 42.

35 Münchener Kommentar/Mayer-Maly, \$138, Rdn.56; Staudinger/H.Dilcher, \138, Rdn. 93; Medicus (o. A. 5), Rdn. 701.

36 Vgl. Honsell (o. A. 14), S.S5.

37 So etwa bei der Diskussion der Frage, ob $₫ 817,2$ BGB auch auf den Vindikationsanspruch anwendbar ist. Das wird mit dem Argument bejaht, es könne nicht angehen, daß bei besonders schweren Sittenverstößen der Anspruch aus $\$ 985$ BGB durchgreifen soll, während bei leichteren, nur auf das Verpflichtungsgeschäften beschränkten Verstößen die Rückforderung ausgeschlossen sein soll. Vgl. in diesem Sinne z. B. Staudinger/Lorenz, $\$ 817$, Rdn. 14; Larenz (o. A. 13), $\$ 69$ III b.

38 Dies die seit RGZ 48, 114 (124); 55, 367 (373); 79, 415 (418); 80, 219 (221) altbekannte, immer wiederholte und abgeschliffene, allerdings zu einem guten Teil gerade erst dadurch zu der Leerformel, als die sie oft bezeichnet wird, herabgestufte Wendung. Vgl. zur Anstandsformel ausführlich Haberstumpf, Die Formel vom Anstandsgefühl aller billig und gerecht Denkenden in der Rechtsprechung des Bundesgerichtshofs, 1976.

39 Dieser Gedanke (u. a.) liegt der in-pari-turpitudine-Regel zugrunde.

40 Ebenso entzieht sich m. E. auch die Fixierung einer exakten Grenze, ab wann etwa anstößig-übermäßige Verträge sittenwidrig sind, rechtswissenschaftlicher Beurteilung und widerspricht im übrigen auch dem Wesen der umfassenden Sittenwidrigkeitsprüfung; hierzu Zimmermann (o. A. 4), S. $47 \mathrm{ff}$., $192 \mathrm{f}$.

${ }_{41}$ Honsell (o.A 14), S. 32 m.w. N.

42 Vgl. nur Motive, in: Mugdan III (o.A. 6), S.5

43 Nicht überzeugend ist die Argumentation von Kegel, Verpflichtung und Verfügung, in: Festschrift für F.A. Mann, 1977, S.77: es sei nicht einzusehen warum, wenn die Verpflichtung sittenwidrig ist, die Erfüllung "sittlich neutral“" sein solle: solle die Verpflichtung zum Mord unsittlich, der Mord aber sittlich neutral sein? Dies Beispiel beweist nichts, da ein Mord keine Verfügung ist.

44 Vgl. z. B. BGH LM $\$ 138$, Nr. 7 a (Aa) und neuerdings etwa BGH NJW 1983, 1851, ferner die Nachweise bei Erman/Brox, \$138, Rdn. 33; Münchener Kommentar/Mayer-Maly, $\$ 138$, Rdn. 11; Palandt/Heinrichs, $\$ 138,1 \mathrm{c}$ bb; Soergel/Hefermebl, \$138, Rdn. 18. 
stab der Inhaltssittenwidrigkeit ${ }^{45}$ : Zuwendungszweck (und erst recht: Motiv) sind irrelevant.

Man mag das Abstraktionsprinzip für rechtspolitisch sinnvoll oder verfehlt ${ }^{46}$ halten. Es ist kein logisch notwendiger Baustein eines Privatrechtssystems. Hat eine Rechtsordnung den Grundsatz aber einmal anerkannt, kann sie nicht nach Belieben damit umspringen, sondern muß ihn auch durchführen. Dies sollte vorliegend auch vom praktischen Resultat her um so leichter fallen, als die Bedeutung des Abstraktionsprinzips viel geringer ist als häufig angenommen wird. Darauf hat erst kürzlich wieder Medicus nachdrücklich hingewiesen ${ }^{47}$. Nur bei „Strafe der Irrationalität und Sinnlosigkeit ihrer eigenen Rede ${ }^{\text {48 }}$ kann sich eine Rechtsordnung, hat sie ein derartiges Prinzip einmal eingeführt, hernach im Einzelfall darüber hinwegsetzen.

\section{'Die Anerkennung von Vollstreckbarerklärung ausländischer Zivilurteile und Schiedssprüche in Kenia}

\author{
Von Rechtsanwalt Professor Dr. Rolf A. Schütze, \\ Stuttgart
}

\begin{abstract}
„Rechtssystem in Kenia - Wirkungserstreckung ausländischer Zivilurteile - Erfordernisse der Wirkungserstreckung ausländischer Zivilurteile - Verfahren der Wirkungserstrekkung ausländischer Zivilurteile - Action upon the Foreign Judgment - Verbürgung der Gegenseitigkeit im Verhältnis zu Kenia gemäß $\$ 328$ Abs. 1 Nr. 5 ZPO - Wirkungserstreckung ausländischer Schiedssprüche in Kenia“.
\end{abstract}

Kenia hat in weiten Bereichen des Rechts common law rezipiert ${ }^{1}$. Gerichtsverfassung und Zivilprozeß folgen dem englischen Mutterrecht ${ }^{2}$.

Das englische Recht stellt zwei Verfahren der Wirkungserstreckung ausländischer Zivilurteile zur Verfügung?:

Die Registrierung von Zahlungsurteilen bei verbürgter Gegenseitigkeit aufgrund besonderen Gesetzes und die Geltendmachung nach common law durch action upon the foreign judgment, soweit eine Registrierung nicht in Betracht kommt.

Grundlage für die Registrierung ausländischer Zivilurteile $^{4}$ war bei Erlangung der Unabhängigkeit zunächst der Foreign Judgments Enforcement Act ${ }^{5}$, dessen Teil IV, der die Anerkennung und Vollstreckbarerklärung von Urteilen aus Nicht-Commonwealth-Staaten zum Gegenstand hat, dem englischen Foreign Judgments (Reciprocal Enforcement) Act, $1933^{6}$ nachgebildet war. Der Foreign Judgments (Reciprocal Enforcement) Act, 1984 hat die bisherige Regelung abgelöst und trägt in vielerlei Hinsicht den Bedürfnissen sich wandelnder rechtlicher Entwicklungen Rechnung.

\section{Erfordernisse der Anerkennung und Vollstreckbarer- klärung}

\section{(1) Zivil-oder Handelssache}

Das ausländische Urteil muß eine Zivil- oder Handelssache zum Gegenstand haben. Auf die Zugehörigkeit des Erstgerichts zu einem bestimmten Gerichtszweig kommt es nicht an. Entscheidend ist allein der Streitgegenstand. Deshalb sind auch Adhäsionsurteile anerkennungsfähig (sect. 3 [1] [c] $)^{8}$. Auch Unterhaltstitel unterfallen dem
Geltungsbereich des Act (sect. 3 [1] [b]). Dies gilt auch für Kostentitel (sect. 3 [1] [e]). Ausgeschlossen aus dem Geltungsbereich des Act sind Urteile in Steuer- und Strafsachen; darüber hinaus auch Entscheidungen, die zu mehrfachem Schadensersatz aus Strafgründen verurteilen (sect. 3 [3] [b]). Diese neue Bestimmung soll die Anerkennung und Vollstreckbarerklärung von Urteilen verhindern, die - wie im US-amerikanischen Recht beispielsweise bei Produkthaftpflichtklagen ${ }^{9}$ dem Geschädigten - auch dem deutschen Recht fremde - Schadensersatzbeträge über den erlittenen Schaden hinaus zusprechen (punitive damages) ${ }^{10}$.

Eine andere Streitfrage löst der neue Act positivrechtlich. Sect. $3[3][\mathrm{m}]$ schließt ausländische Exequatururteile von der Wirkungserstreckung aus. Es gibt also keine Doppelexequierung ${ }^{11}$ eines ausländischen Urteils. Das Recht Kenias hat sich zugunsten des Prinzips: „L'exequatur sur l'exequatur ne vaut " ${ }^{12}$ entschieden. Ausgenommen aus dem sachlichen Anwendungsbereich des Act sind schließlich Entscheidungen in gewissen familien- und erbrechtlichen Materien.

\section{(2) Zablungs- oder Herausgabeanspruch}

Das ausländische Urteil muß zur Zahlung oder Herausgabe einer beweglichen Sache verurteilen. Die

45 Anders ausdrücklich Münchener Kommentar/Mayer-Maly, \$138, Rdn. 138 a. E.

46 So neuerdings v. a. Kegel (o. A. 43), S. 57 ff.; abgewogen Baur (o. A. 17), $\$ 51$ VIII 1; Flume (o. A. 3), $\$ 12$ III 3: Medicus (o.A. 5), Rdn. 230

${ }_{47}$ Medicus (o. A. 5), Rdn. $227 \mathrm{ff}$. Ein Beispiel ist die praktisch bedeutungslose Diskussion um die Frage, ob das Erfüllungsgeschäft des Wucherers wirksam ode unwirksam ist. Er verliert das Eigentum sowieso in aller Regel durch Vermischung.

48 Hruschka, Strafrecht nach logisch-analytischer Methode, 1983, S. XI

1 Vgl. dazu Cotran, Länderbericht Kenia: International Encyclopedia of Comparative Law, Bd.I, K - $1 \mathrm{ff}$; ders., Kenya bei: Allott u. a. Judicial and Lega Systems in Africa, 2. Aufl., 1970, S. 132 ff., Rogers, Commercial Law in Eas Africa, 1969; Zweigert/Kötz, Einführung in die Rechtsgleichung, 1971, Bd.I, S. $271 \mathrm{ff}$

Vgl. dazu Braune, Die Rechtspflege in den britischen Gebieten Afrikas, 1941 (veraltet, aber noch lesenswert); Spry, Civil Procedure in East Africa, 1969.

$3 \mathrm{Vgl}$ dazu Borm/Reid, The Recognition and Enforcement of Foreign Judg ments in England: IntComp. L. Q. 3 (1954), S. 49 ff.; Read, Recognition and Enforcement of Foreign Judgments in the Common Law Units of the British Commonwealth, 1938; Geimer/Schütze, Internationale Urteilsanerkennung, Bd. II, 1971, S. 355 ff. m. w. N.

${ }^{4}$ Vgl. zum bisherigen Rechtszustand Martiny, Anerkennung ausländischer Entscheidungen nach autonomem Recht: Handbuch des Internationalen Zivilverfahrensrechts, Bd. III, 1, 1984, Rdn. 1415 f.; Schütze, Vollstreckung ausländischer Urteile in Afrika, 1966, S. 35 f.; ders., Die Geltendmachung deutscher Urteile im Ausland - Verbürgung der Gegenseitigkeit, 1977, S. 63 ff.; ders., Die Anerkennung und Vollstreckung ausländischer Zivilurteile in Ostafrika: Monatsschrift der Vereinigung deutscher Auslandsbeamten 1964, S. $293 \mathrm{ff}$.

5 Cap. 43 of the Laws of Kenya.

$623 \mathrm{Geo} . \mathrm{V}, \mathrm{c} .13$.

7 Act 4/1984, veröffentlicht in Kenya Gazette Supplement Nr.28 v. 18.5.1984.

8 Vgl. zur Problematik der Anerkennung von Adhäsionsurteilen allgemein die gegensätzlichen Gutachten von Kohlrausch und Pagenstecher: Kann ein in Abwesenheit des Angeklagten ergangenes Adhäsionsurteil des Zürcherischen Schwurgerichtshofes in Deutschland für vollstreckbar erklärt werden?: RheinZ Bd.12, S. $129 \mathrm{ff} ., 139 \mathrm{ff}$

9 Vgl. dazu von Westphalen, „Punitive Damages“ in US-amerikanischen Produkthaftungsklagen und der Vorbehalt des Art.12 EGBGB: RIW/AWD 1981, S. $141 \mathrm{ff}$.

${ }_{10}$ Die Anerkennungsfähigkeit von Entscheidungen, die zu punitive damage verurteilen, ist in der deutschen Lehre umstritten. Gegen eine Anerkennungsfähig keit haben sich ausgesprochen von Hülsen, Produkthaftpflicht USA 1981: RIW/ AWD 1982, S. 1 ff. (9) und HoechstVersR 1983, S. 14 ff. (16 f.), dafür von Westphalen RIW/AWD 1981 S.141 ff.; Martiny, Anerkennung ausländischer Entscheidungen nach autonomem Recht: Handbuch des Internationalen Zivilverfahrensrechts, Bd. III/1 1984, Rdn. 507 differenziert. Soweit durch punitive damages nach allgemeinen Regeln nicht ersatzfähige Vermögensschäden abgedeckt werden, sol die Entscheidung anerkennungsfähig sein, nicht jedoch, wenn der Strafzweck im Vordergrund steht.

11 Vgl. dazu Schütze, Die Doppelexequierung ausländischer Zivilurteile: ZZP 77 (1964), S. $287 \mathrm{ff}$

12 Nach der Terminologie von Gavalda, Journal Clunet 135, S.135 und Malaurie, Journal Clunet 1970, S.941. Der Grundsatz wird im deutschen Rech von der h. L. favorisiert, z. B. von Geimer, Zur Prüfung der Gerichtsbarkeit und der internationalen Zuständigkeit bei der Anerkennung ausländischer Urteile, 1966, S. 26 Fn. 7 und Zöller-Geimer, ZPO, 14. Aufl., $\$ 328$ Rdn. 86 m.w.N. 